\title{
A Study on Academic Influence of the Chinese Translation Version of On the Philosophy of Higher Education by John S. Brubacher
}

\author{
Fengmin Yu \\ Scientometrics Research Center, Zhejiang Shuren University, Hangzhou, China
}

\section{Email Address:}

Yufomy@qq.com

To cite this article:

Fengmin Yu. A Study on Academic Influence of the Chinese Translation Version of On the Philosophy of Higher Education by John S. Brubacher. International Journal of Education, Culture and Society. Vol. 3, No. 3, 2018, pp. 53-60. doi: 10.11648/j.ijecs.20180303.13

Received: June 7, 2018; Accepted: September 29, 2018; Published: October 29, 2018

\begin{abstract}
Using bibliometrics, this study analyzes the articles citing the Chinese translation of John S. Brubacher's seminal text, On the Philosophy of Higher Education. The purpose and significance of this study is: through a comprehensive description of the academic influence of the Chinese version in China, to provide research hotspots and shortcomings for the researchers in this area, to seek scientific cooperation with the core authors, to provide reference for building and perfecting the philosophy system of higher education. So, this study includes a discussion of their academic influence by year, source, author and institution; and finds that the book has become a time-honored research classic cited or elaborated or questioned by scholars of higher education in China, Huazhong University of Science and Technology is greatly influenced by the Chinese translation version, and Wang Jianhua from Xiamen University Higher Education Institute is much more affected than other authors. In addition, this paper examines the high frequency keywords and their co-occurrence in these citing articles to investigate the hotspots of academic influence and focus of this influential book. The hotspots of citing articles mainly include academic freedom, university autonomy, general education, professional education, university culture, value orientation, education fairness, administrative power, government, university organization, professorship, liberal education, etc. A content analysis is used as well to sort the main ideas of his research and evaluate the impact of On the Philosophy of Higher Education in China.
\end{abstract}

Keywords: John S. Brubacher, On the Philosophy of Higher Education, Academic Influence, Citing Article

\section{Introduction}

John S. Brubacher (1898-1988) was a famous scholar in the field of higher education in the 20th century. Many of the classic monographs on higher education were written by Brubacher, such as Higher Education in Transition: an American History, Bases for Policy in Higher Education, On the Philosophy of Higher Education, etc. Among these books, On the Philosophy of Higher Education has become a classic in the field of higher education. "It is a famous academic monograph recommended by ACRL for his scientific research on the comprehensive and in-depth study of the higher education", "The author has constructed a complete philosophical system of higher education" [1]. The book cited by scholars widely, the academic position is beyond doubt.

In this book, Brubacher used philosophical theory to answer various problems that have arisen in the development of higher education since World War II. Based on the current status of higher education in the United States at that time, he put forward eight basic questions about higher education, that is "The Higher Learning", "Academic Autonomy", "Academic Freedom", "Higher Education for Whom?", "General and Specialized Education", "Pedagogy of Higher Education", "Ethics of Scholarship", and "The University as a Church" [2].

Since the Chinese translation version of On the Philosophy of Higher Education publishing in 1987, its interpretation, research and debate have been very prevalent. The influence on China's higher education is significant. The book provides resources and samples for imitation and reference for higher education researchers in China. The Chinese translation version has an increasingly profound impact on China's higher education disciplines, theoretical research, and practice [3].

There are two versions of the Chinese translation of the 
book On the Philosophy of Higher Education. One is the version of Zheng Jiwei as the first translator in the 1987 edition. This version was revised and reprinted in 1989 and 1998; the other version was Wang Chengxu as the first one published in 2002. Both versions were published by Zhejiang Education Press, and the scholars involved in the translation were actually five people including Zheng Jiwei, Wang Chengxu, Zhang Weiping, Xu Hui, and Zhang Minxuan of Zhejiang University (formerly Hangzhou University), but the first translator has exchanged.

Both Chinese translations have higher influence in Zhejiang edition books in China. By 2006, Zheng's version was cited 3702 times, and ranked first in all Zhejiang edition books; Wang's was cited 1536 times, ranking fifth [4].

\section{Research Status, Research Methods and Samples}

\subsection{Literature Review}

In these studies cited the Chinese version of the book, there are some voices of questioning and introspection. Some scholars believed that the accuracy of "The Higher Learning" as a logical basis of higher education philosophy and the accuracy of the book as "a complete philosophy system of higher education" are not worth continuing to explore [5]. Other scholars believed that it is not enough to use "The Higher Learning" as the basis of philosophy of higher education; it's not necessary to attempt to coordinate epistemology and politics, there is no clear limit to academic autonomy and academic freedom, vocational education is not just professional education to learn special knowledge [6].

Through the quantitative statistical analysis of literature cited in the work (thesis), one can understand the academic influence of the work (thesis), and can also ascertain the most influential work or author in a particular subject area $[7,8]$. With regard to the study of the academic influence of the Chinese version of "On the Philosophy of Higher Education", there have been some achievements in China, but more are qualitative comments, and the number of academic papers combined with quantitative research is small.

Song Nana (2012) used the CSSCI (Chinese Social Sciences Citation Index) as the research object and interpreted articles that cited the Chinese version of the book; believed that due to the differences in the English and Chinese contexts and the differences between the Chinese and American cultures, that produced a certain degree of misreading (mistranslation) [9]. Another paper that compares the number of articles cited in the Chinese version in the journal of Higher Education collected by Information Center for Social Sciences of Renmin University of China, and the number of articles in the domestic review of the book, and divides the introduction of "On the Philosophy of Higher Education" in china into three stages: the introduction as a reference book(1987-1998), the introduction as a commentary (1999-2003), and the introduction as criticism and reflection (2004-present) [10].

\subsection{Research Questions and Purpose}

So, what exactly is the academic influence of the Chinese version of On the Philosophy of Higher Education? This paper attempts to answer some questions from the point of view of the book being cited:

Who (author, institution) cited the Chinese version book?

Which year did the citing articles cite the Chinese version book?

Which type of article did cite the Chinese version book?

What did citing articles say about the Chinese version book?

Therefore, the purpose and significance of this study are as follows: Through a comprehensive description of the academic influence of the Chinese version in China, to provide research hotspots and shortcomings for the researchers in this area, to seek scientific cooperation with the core authors, to provide reference for building and perfecting the philosophy system of higher education.

\subsection{Research Methods and Samples}

The research methods adopted in this paper mainly include "bibliometrics" and "content analysis", and the combination of qualitative and quantitative methods is used to investigate the academic influence of the Chinese version of "On the Philosophy of Higher Education".

The research samples of this paper are sourced from the China Knowledge Network Database Resource (www.cnki.net). The database includes more than 8,000 Chinese academic journals, much of international outstanding academic conferences, and much amount of master's degree dissertations. Its search function is very comprehensive.

The specific approach is: In the advanced search of the CNKI homepage, select "References" as the search item and the search content is "Brubacher and On the Philosophy of Higher Education". A total of 9148 citing articles of Brubacher's book were retrieved (retrieve time: 2018/5/10). There are 5685 journal articles, 3391 dissertations, and 72 conference articles. Due to the small number of conference articles, only used journal articles and dissertations as research samples, totaling 9076 articles.

\section{Academic Influences}

\subsection{Distribution Characteristics of Citing Articles}

\subsubsection{Annual Distribution}

More than 9076 articles have cited the Chinese version publication of Brubacher's "On the Philosophy of Higher Education" since it published in 1987. Figure 1 shows the annual distribution of citing articles from 1987 to the present. Find that the earliest citing article of "On the Philosophy of Higher Education" is a journal article with the title of "University Professor and University Education Policy", published on the journal of "Advanced Engineering Education Research" in 1998. It quoted to the view of political theory in "On the Philosophy of Higher Education" when discussing the 
role and role of university professors in formulating university policies but not neglecting the leadership role of government and university administration [11].

By 1992, there was a citing article of the book every year. Since 1993, the number of citing articles has gradually increased, and has increased sharply after 1999; the total amount of citing articles peaked in 2007 and 2008. After 2009, the number has declined, and has dropped significantly in the past two years.

From the types of citing articles, the number of journal article was more than the number of dissertation in each year; the number of journal article remained at more than 400 in 2007-2013, and 2015; the trend of dissertation number was basically consistent with that of the journal article.

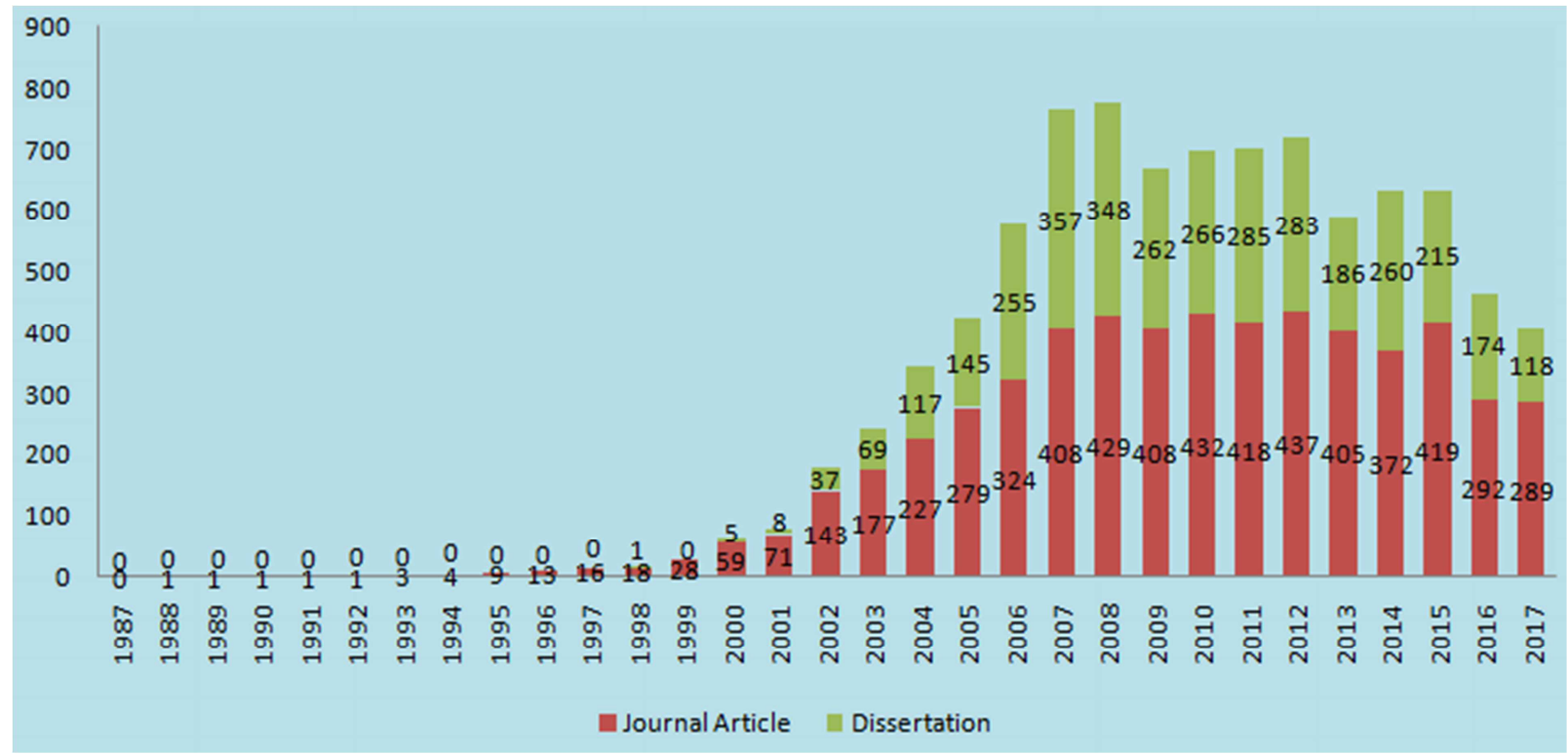

Figure 1. Annual distribution of citing articles.

\subsubsection{Source Distribution}

There are 1,528 sources of citing articles (5.9 articles per source), of which 1272 are Chinese journals (4.5 articles per journal), 256 are degree-award institutions (13.2 articles per inst.), and the citing articles are more concentrated in the dissertations of universities. Table 1 and table 2 list the degree-award institutions and journals with 30 or more citing articles that can basically reflect the distribution of the academic influence of the Chinese version of "On the Philosophy of Higher Education".

The book has a great influence on the dissertations of East China Normal University, Southwest University, Huazhong University of Science and Technology, Hunan Normal University, and Central China Normal University (Table 1). In particular, East China Normal University has a high number of 201 articles, far more than the number of citing articles in the journal of "Higher Education Studies" with the largest number of journal articles (Table 2). From the statistics of dissertations in CNKI, there are 1421 dissertations in the higher education disciplines of East China Normal University (date of search: 2018/05/11), and 14.1\% of dissertations cite the Chinese translation version.

Table 1. Main Sources Distribution (for dissertations).

\begin{tabular}{ll}
\hline Degree-award institution & Number of citing articles \\
\hline East China Normal University & 201 \\
Southwest University & 199 \\
Huazhong University of Science and & 180 \\
\hline
\end{tabular}

\begin{tabular}{ll}
\hline Degree-award institution & Number of citing articles \\
\hline Technology & 155 \\
Hunan Normal University & 109 \\
Huazhong Normal University & 82 \\
Suzhou University & 79 \\
Shanxi University & 75 \\
Shandong Normal University & 74 \\
Hunan University & 73 \\
Central South University & 73 \\
Jilin University & 69 \\
Northeast Normal University & 67 \\
Wuhan University of Technology & 66 \\
Nanjing Normal University & 51 \\
Xiamen University & 49 \\
Jiangxi Normal University & 48 \\
Fujian Normal University & 48 \\
Guangxi Normal University & 44 \\
Zhejiang Normal University & 44 \\
Hebei University & 44 \\
Hohai University & 44 \\
Northwest Normal University & 39 \\
South-Central University for Nationalities & 39 \\
Xiangtan University & 38 \\
Sichuan Normal University & 38 \\
Lanzhou University & 38 \\
Shan Dong University & 36 \\
Qufu Normal University & 36 \\
Henan University & 35 \\
Shaanxi Normal University & 33 \\
Shenyang Normal University & \\
\hline
\end{tabular}


Table 2. Main Sources Distribution (for journal articles).

\begin{tabular}{|c|c|}
\hline Journal name & Number of citing articles \\
\hline Higher education research & 187 \\
\hline Jiangsu Higher Education & 167 \\
\hline Heilongjiang Higher Education Research & 124 \\
\hline Modern university education & 120 \\
\hline Modern Education Science & 111 \\
\hline Higher Education Exploration & 101 \\
\hline China Higher Education Research & 98 \\
\hline Journal of National Academy of Education Administration & 92 \\
\hline University Education Science & 91 \\
\hline Meitan Higher Education & 89 \\
\hline Modern education management & 80 \\
\hline Tsinghua Journal of Education & 69 \\
\hline Research in Educational Development & 67 \\
\hline Fudan Education Forum & 59 \\
\hline Educational Research & 56 \\
\hline Higher Agricultural Education & 53 \\
\hline Journal of Higher Education Management & 52 \\
\hline Research in Higher Education of Engineering & 52 \\
\hline Cultural and educational information & 49 \\
\hline China Electric Power Education & 45 \\
\hline Education and Vocation & 43 \\
\hline Academic Degrees \& Graduate Education & 42 \\
\hline China adult education & 41 \\
\hline Educational exploration & 41 \\
\hline Higher Education of Science & 40 \\
\hline Education Review & 37 \\
\hline International and Comparative Education & 36 \\
\hline Modern Education Management & 36 \\
\hline Design Research & 34 \\
\hline Journal of Educational Science of Hunan Normal University & 32 \\
\hline Journal of Yangzhou University (Higher Education Study Edition) & 31 \\
\hline Journal of Inner Mongolia Normal University(Educational Science) & 30 \\
\hline
\end{tabular}

\subsubsection{Author Distribution}

There are 6,266 first authors in total of 9076 citing articles, that is, each author published 1.4 articles that cited the Chinese version of "On the Philosophy of Higher Education". Table 3 is the names of the first authors who published 10 or more citing articles. These authors are deeply influenced by the Chinese translation. Among them, Wang Jianhua from the Institute of
Higher Education of Xiamen University publishes the most number of citing articles, far more than other authors. The authors in table 3 are all from university higher education institutes or related colleges, even university leaders. According to "Price's Law", it can be calculated that the core authors should have 5 articles or more. Therefore, there are as many as 175 core authors who are most affected by the Chinese translation version.

Table 3. Main Authors Distribution of the Citing Articles.

\begin{tabular}{llll}
\hline Author Name & Number of citing articles & Author Name & Number of citing articles \\
\hline Wang Jianhua & 31 & Li Xiaoying & 11 \\
Gong Fang & 20 & Liu Zhentian & 11 \\
Xuan Yong & 16 & Wang Enhua & 11 \\
Liu Xin & 16 & Peng Yongjun & 11 \\
Sheng Zhengfa & 15 & Hu Rendong & 11 \\
Li Zhifeng & 15 & Jiang Hongchi & 11 \\
Chen Wei & 15 & Li Yunqing & 11 \\
Li Shuohao & 14 & He Shutong & 11 \\
Sui Yifan & 13 & Wang Fuyou & 10 \\
Zhou Guangli & 13 & Shao Bo & 10 \\
Bie Dunrong & 13 & Zuo Chongliang & 10 \\
Fang Zeqiang & 13 & Ma tingqi & 10 \\
Fan Huaqiang & 12 & Zhao Jun & 10 \\
Xiang Dongchun & 12 & Liu Fanfeng & 10 \\
Yang Kerui & 12 & Ma Fengqi & 10 \\
Chen Hefang & 12 & Guo Feng & 10 \\
Zhu Weihong & 12 & Zhu Xiaogang & 10 \\
Wang Fei & 11 & Zhang Yizhong & 10 \\
Zhang Chuting & 11 & Li Jianhui & 10 \\
\hline
\end{tabular}




\subsubsection{Institution Distribution}

According to the statistics of the institution of the first author, Huazhong University of Science and Technology, East China Normal University, Southwest University, Xiamen University, and Hunan Normal University published more than 200 citing articles. Table 4 lists 41 institutions with 50 or more citing articles. Undoubtedly, the Chinese translation version of "On the Philosophy of Higher Education" has the greatest influence on these institutions. These institutions are all from universities, of which China key universities account for the vast majority, while normal universities account for nearly $40 \%$.

Table 4. Main Institutions Distribution of the Citing Articles.

\begin{tabular}{ll}
\hline Institution Name & Number of citing articles \\
\hline Huazhong University of Science and & 432 \\
Technology & 286 \\
East China Normal University & 258 \\
Southwest University & 245 \\
Xiamen University & 229 \\
Hunan Normal University & 176 \\
Nanjing Normal University & 175 \\
Huazhong Normal University & 145 \\
Zhejiang University & 136 \\
Nanjing University & 135 \\
Suzhou University & 115 \\
Wuhan University of Technology & 115 \\
Central South University & 112 \\
Hunan University & 111 \\
Shanxi University & 105 \\
Zhejiang Normal University & 100 \\
Jilin University & 99 \\
Beijing Normal University & 95 \\
South China Normal University & 86 \\
\hline Jiangxi Normal University &
\end{tabular}

\begin{tabular}{ll}
\hline Institution Name & Number of citing articles \\
\hline Shandong Normal University & 85 \\
Shaanxi Normal University & 82 \\
Northeast Normal University & 81 \\
Guangxi Normal University & 81 \\
South Central University for Nationalities & 80 \\
Hebei University & 78 \\
Hohai University & 73 \\
Peiking University & 73 \\
Lanzhou University & 70 \\
Fujian Normal University & 69 \\
Xiangtan University & 69 \\
Shenyang Normal University & 69 \\
Northwest Normal University & 67 \\
Shantou University & 61 \\
Dalian University of Technology & 60 \\
Henan University & 58 \\
Qufu Normal University & 56 \\
Shan Dong University & 55 \\
Hunan Agricultural University & 54 \\
Zhejiang University of Technology & 53 \\
Shanghai Normal University & 52 \\
Fudan University & 51 \\
\hline
\end{tabular}

\subsection{Content Analysis}

\subsubsection{Keyword Frequency}

The content analysis of citing articles can understand the main manifestations of academic influence of the Chinese translation version of "On the Philosophy of Higher Education". The keywords are the vocabulary that the author can best reflect the content of his paper. Table 5 lists the 47 keywords whose frequency is 50 times or more in 9076 articles.

Table 5. High-frequency Keywords of Citing Articles.

\begin{tabular}{llll}
\hline Keyword & Frequency & Keyword & Frequency \\
\hline High Education & 830 & University Culture & 91 \\
University & 622 & Reform & 87 \\
Colleges and Universities & 533 & John S. Brubacher & 82 \\
Academic Freedom & 352 & Professional Education & 80 \\
Academic Power & 250 & Value Orientation & 79 \\
College Students & 202 & Postgraduate Education & 75 \\
General Education & 200 & Vocational College & 73 \\
University Autonomy & 174 & University Teachers & 71 \\
Education Fair & 171 & Modern University & 71 \\
Countermeasures & 170 & Development & 69 \\
Administrative Power & 168 & Private colleges and universities & 66 \\
United States & 156 & Idea & 64 \\
University Spirit & 153 & Postgraduate & 63 \\
Modern University System & 147 & Ideological and Political Education & 63 \\
University Philosophy & 125 & Culture & 62 \\
University Teachers & 121 & De-administration & 62 \\
On the Philosophy of Higher Education & 120 & Popularization & 62 \\
University Governance & 111 & Innovation & 62 \\
Government & 103 & System & 62 \\
Research Universities & 97 & Course & 61 \\
Enlightenment & 95 & Teaching & 61 \\
Talent Development & 93 & Local Colleges and Universities & 61 \\
Problem & 92 & University Regulations & 60 \\
\hline
\end{tabular}

In table 5, "university" and "higher education" are the main objects of these citing article studies, and the contents of research are mostly contained in the "eight basic questions" proposed by "On the Philosophy of Higher Education", such 
as "Academic Freedom", "University Autonomy", "General Education", "Professional Education", etc.

\subsubsection{Keywords Co-Occurrence}

If two keywords appear together in different articles (keywords co-occurrence) several time, then the topics related to these two keywords are considered to be the focus of the researchers. The keywords co-occurrence can be intuitively displayed using the method of knowledge mapping. Figure 2 shows the keywords co-occurrence knowledge mapping of the high-frequency keywords of citing articles drawn by PAJEK (co-occurrence times $>=10$ ). The dot size indicates the level of keyword frequency, and the line thickness indicates the number of co-occurrences.

In figure 2, the focus of the citing articles research is mainly on the balance between "administrative power" and "academic power", "general education" and "professional education" in universities; how to deal with the relationship between "University" and "Government", "Academic Autonomy" and "Academic Freedom". The popularization of higher education is also highly concerned with the citing articles; of course, the evaluation and research on Brubacher's book is also a focus on.

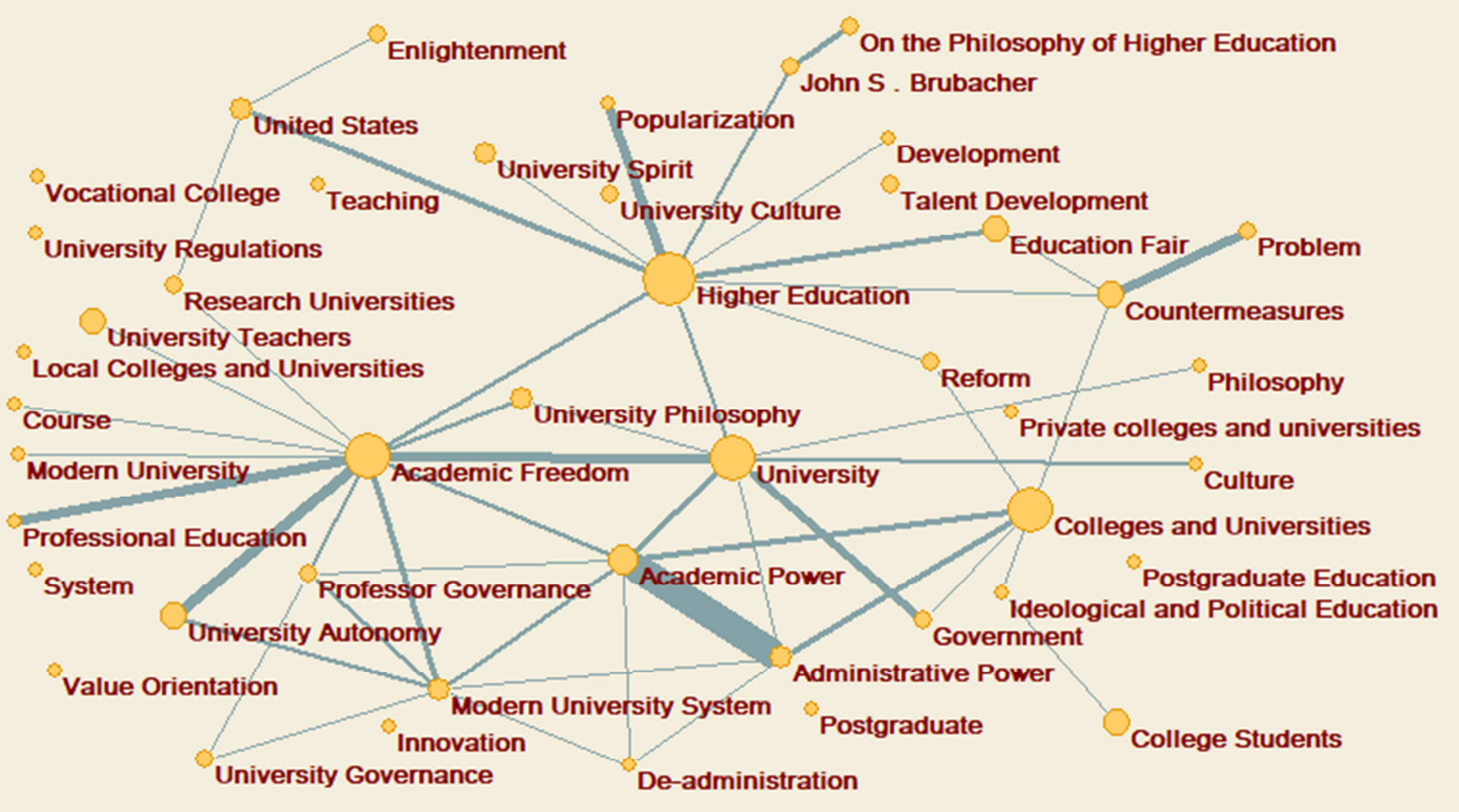

Figure 2. A Knowledge Mapping of High-frequency Keywords.

\subsubsection{Main Content and Viewpoint}

\section{(1) Interpretation}

In the early period of the publication of the Chinese version of "On the Philosophy of Higher Education", Chinese scholars stayed for a long time in its interpretation. such as professor's knowledge structure, professional ethics and teaching arts , "high learning" and its caregivers and guardians , combination of general education and professional education, and opposition between two values of higher education and the opposition, conflict and coordination of university philosophy [15], university service functions [16], academic freedom [17], the value of knowledge [18], the university of the church [19], the science of higher education [20], etc. These studies all include the "eight questions" proposed by Brubacher.

(2) Revelation and Insight

Since the background of the book "On the Philosophy of Higher Education" is the 20th century in the United States, its main research object is American higher education, and there are the differences in Chinese and American culture and history. Therefore, many viewpoints cannot be completely transplanted to China [21]. In the process of interpreting the Chinese translation version, many scholars believe that after understanding and absorbing its ideas have important implications for the development of modern university education in China. In particular, with the expansion of the autonomy of colleges and universities in China, reforms in personnel training and curriculum structure are needed. Brubacher's thoughts on college curriculum development and curriculum design have important implications for the reform of university curriculum management in China [22, 23].

Another example is Brubaker's viewpoint of "personal autonomy". Some scholars believe that the essence of the "Ma Jiajue" incident in 2004 was the loss of "personal autonomy" [24]. The philosophical viewpoint of higher education in China's "political theory" has always occupied a major position. The ideas of "government standard", "scientific only", "technical only", "little humanities", "light moral 
education" are serious. As time goes by, Brubacher's revelations about the university's function, academic freedom, and educational equity bring us particularly important insights [25]. The higher education in China needs to consider deeply from the level of philosophy of higher education and construct a philosophical system of higher education with Chinese characteristics [26].

(3) Question and Reflection

All along, scholars in China have studied Philosophy in the field of higher education based on the book of "On the Philosophy of Higher Education", and there is also a certain amount of research dependence. Until 2005, some scholars have questioned and introspect it. Yang Min used the starting point of "Does the philosophy of higher education need to build a strict logical system?" and profoundly rethought "higher education", "political theory" and "knowledge monopoly" [27]. Li Zhifeng considered that the book has three defects: there are limitations on the relationship between political theory and epistemological philosophical view; its philosophical concept ignores the human-centered theory; the philosophy of higher education can be handled as a whole, in fact, the conflict and coexistence of the philosophical view of modern western higher education is the main reason for promoting the diversified development of higher education [28].

Some scholars have questioned Brubaker's philosophical foundation of higher education political theory and epistemology, arguing that it "cannot cover all the legitimacy of the existence of higher education, and the reason why higher education exists and its more profound original foundation - The need for high-level development of people, "[29] Human being are the philosophical foundation and the common basis for higher education, and also the starting point and destination of higher education reform and development." The practice of higher education reform and development urgently requires the return of philosophy of higher education to people; promote students and teachers to "plan" and "go beyond" individual life to achieve the growth and development of social roles, so as to ensure the continuation and development of social and human civilization [31]. Philosophy of higher education should adhere to the issue of priority and grasp the requirements of the times [32].

Yang Xingfang made a comprehensive criticism, reflection, and summary of the malformation study of China's higher education philosophy in the sorrowful title of "30 years of reading and 3 years of awakening" [33]. Thought that "On the Philosophy of Higher Education" had been put in a wrong place from the book translated beginning. One of the main reasons for this book is that the scholars equate the "system of philosophical studies on higher education" based on Brubacher's "The Higher Learning" with the "philosophical system of higher education" and the "discipline system of philosophy of higher education".

\section{Conclusions}

Based on the above analysis, some conclusions can be drawn roughly:
From the annual distribution of citing articles, the academic influence of Brubaker's Chinese translation has rapidly increased since 1999, peaked in 2007-2008, and continued until 2015, and a slight decline in the past two years. The book has become a time-honored research classic cited or elaborated or questioned by scholars of higher education in China.

The authors who are greatly influenced by the Chinese translation version are all from higher education institutes or relevant departments of the national key universities, such as Huazhong University of Science and Technology, East China Normal University, Southwest University, Xiamen University, and Hunan Normal University. Wang Jianhua from Xiamen University Higher Education Institute is much more affected than other authors. The contents of citing articles mainly include academic freedom, university autonomy, general education, professional education, university culture, value orientation, education fairness, administrative power, government, university organization, professorship, liberal education, etc.

Unfortunately, the source of the study sample was flawed in this study. Although the CNKI is relatively comprehensive in the collection of journal papers, not comprehensive in dissertations. In particular, dissertations from top universities in China, such as Peking University, Tsinghua University, etc. are not included.

\section{References}

[1] J. S. Brubacher; translated by Zheng, Jiwei., et al (1987). On the Philosophy of Higher Education. Hangzhou, Zhejiang: Zhejiang Education Press.

[2] J. S. Brubacher (1982). On the Philosophy of Higher Education. San Francisco, CA: Jossey-Bass.

[3] Liu Yamin, Hou Huaiyin (2011). The Introduction and Influence of Brubacher's "On the Philosophy of Higher Education". in China. Heilongiang Higher Education Research, 4: 50-53.

[4] Yu Fengmin, Lin Yanru (2013). Characteristics of Zhejiang academic development and achievements since the founding of the People's Republic of China: based on cited analysis of Zhejiang edition books (1950-2006). Journal of Library and Information Science in Zhejiang Province, 4: 16-22.

[5] Wu Di (2010). Review and reflection on Brubacher's "On the Philosophy of Higher Education". Journal of Xuzhou Normal University (Philosophy and Social Sciences Edition), 4:134-138.

[6] Song Xiaoping (2009). Reflections on Brubacher 's philosophy of higher education. Journal of Hunan University of Science and Technology, 4:126-128.

[7] $\mathrm{Hu}$ Yue (2009). The most influential foreign academic works on the study of Chinese philosophy - based on CSSCI analysis. Dong Yue Lun Cong, 2:60-67.

[8] Yang Qin (2010). Analysis of the most influential domestic academic works on the study of pedagogy in China: based on CSSCI (2000-2007). Journal of Southwest University for Nationalities, 2:258-270. 
[9] Song Nana (2012). Brubacher's "On the Philosophy of Higher Education" in the Chinese context: an interpretation study based on CSSCI (1998-2010). Modern University Education, 5:19-27.

[10] Liu Yamin, Hou Huaiyin (2011). The Introduction and Influence of Brubacher's "On the Philosophy of Higher Education". in China. Heilongjiang Higher Education Research, 4: 50-53.

[11] Chen Haichun, Yao Qihe (1988). University professors and education policies. Research in Higher Education of Engineering, 4: 17-21.

[12] Shao Haiyan (1996). Philosophical reflections on professor qualification - reading Brubacher's "On the Philosophy of Higher Education”. Teacher Training Research, 4:13-16.

[13] Hu Bicheng (2000). Scholarship should become a way of life in academia: the morality of scholarship of Brubacher's "On the Philosophy of Higher Education". Journal of Central South University of Technology (Social Sciences Edition), 4:323-326.

[14] Liu Chengbo (2001). On the professional education and general education in modern universities: a review of John S. Brubacher's "On the Philosophy of Higher Education". Journal of Higher Education Research, 2001(1):26-29.

[15] Zhang Huijie (2001). A masterpiece of higher education values: thinking about Brubacher's "On the Philosophy of Higher Education”. Higher Agricultural Education, 6:10-13.

[16] Qiu Yi (2001). A further discussion on the university's service function: on Brubacher's "On the Philosophy of Higher Education". Building Materials Higher Education Theory and Practice, 5:41-43.

[17] Mi Junkui (2004). Some thoughts on academic freedom: reading John S. Brubacher's "On the Philosophy of Higher Education". Journal of Inner Mongolia Normal University (Education Science Edition), 3:5-7.

[18] Liu Zhiwen (2004). The value of knowledge and philosophy of higher education: a study report on Brubacher's "On the Philosophy of Higher Education". Modern University Education, 4:21-23.

[19] Guo Lijun (2004). On the implication of university as a modern church: an interpretation of Brubacher's "On the Philosophy of Higher Education”. Coal Higher Education, 5:48-50.

[20] Lin Zhi (2005). Interpreting higher education: on Brubacher's "On the Philosophy of Higher Education". Journal of Yunyang Teachers College, 2:104-105.

[21] Li Huaping, Han Yingxiong (2004). Social differences in the development of higher education between China and the U. S. A.: a study of Brubacher's "On the Philosophy of Higher Education". Research in Higher Education of Technology, 4:23-24.
[22] Chen Xingming (2004). Attach importance to the research and management of college curriculum development process: Brubacher's thoughts on university curriculum design and its enlightenment. Yunnan Education, 18:39-41.

[23] Ouyang Wen (2007). University curriculum reform in the perspective of Brubacher's Higher Education Philosophy. Jiangsu Higher Education, 3:81-83.

[24] Xiong Huajun (2004). Education for candidates in "Personal Autonomy": reflection on "Ma Jiajue Case" - Reading Brubacher's "On the Philosophy of Higher Education". Hubei Entrance Examination, 12:56-59.

[25] Peng Huimin (2006). Philosophical reflections on the background of popularization of higher education: a study of Brubacher's "On the Philosophy of Higher Education”. Higher Agricultural Education, 8:69-72.

[26] Yang Denghong (2009). Brubacher's "On the Philosophy of Higher Education" and its enlightenment to us. Journal of Hubei Normal University (Philosophy and Social Sciences), 2: 131-133.

[27] Yang Min (2005). Reflection on philosophy of higher education. Chinese Journal of Metallurgy, 6:35-37.

[28] Li Zhifeng (2006). Rethinking and critique of western higher education philosophical viewpoint - Also on Brubacher's "On the Philosophy of Higher Education”. Modern Educational Science, 9:1-4.

[29] Song Caiping, Wang Liping (2008). Questioning Brubacher's philosophical basis of higher education. Fudan Education Forum, 5:32-35.

[30] Lin Jie (2014). Reflection and reexamination of the philosophical basis of higher education: based on the perspective of humanism theory. Higher Agricultural Education, 6:14-16.

[31] Ma Xiaoyong, Wei Jianjian (2012). On the humanistic foundation of higher education philosophy. Educational Research, 12:29-34.

[32] Cai Zhongbing, Liu Zhiwen (2017). A study of Brubacher's "On the Philosophy of Higher Education", and its Phenomenological Analysis. Journal of Guangzhou University (Social Science Edition), 11:70-75.

[33] Yang Xingfang (2009). "30 Years of Reading and 3 Years of Awakening": On the academic development path of China's higher education philosophy study. Jiangsu Higher Education, 4:7-10. 